\title{
Bisimilarity is not Borel $^{\dagger}$
}

\author{
PEDRO SÁNCHEZ TERRAF \\ CIEM-FAMAF, Universidad Nacional de Córdoba, \\ Medina Allende s/n (Ciudad Universitaria), X5000HUA - Córdoba, Argentina \\ Email: sterraf@famaf .unc.edu.ar, \\ and \\ Algebraische und Logische Grundlagen der Informatik - Institut für Theoretische Informatik \\ Technische Universität Dresden - Fakultät Informatik - 01062 Dresden
}

Received 6 March 2013; revised 6 January 2014

We prove that the relation of bisimilarity between countable labelled transition systems (LTS) is $\boldsymbol{\Sigma}_{1}^{1}$-complete (hence not Borel), by reducing the set of non-well orders over the natural numbers continuously to it.

This has an impact on the theory of probabilistic and non-deterministic processes over uncountable spaces, since logical characterizations of bisimilarity (as, for instance, those based on the unique structure theorem for analytic spaces) require a countable logic whose formulas have measurable semantics. Our reduction shows that such a logic does not exist in the case of image-infinite processes.

\section{Introduction}

Markov decision processes over continuous state spaces are an appropriate framework to study and formalize systems that involve continuously valued variables such as those arising in physics, biology, and economics; and where some of those variables are known only in a probabilistic way.

In this direction, labelled Markov processes (LMP) were developed by Desharnais (1999) and Desharnais et al. (2002). An LMP has a labelled set of actions that encode interaction with the environment; thus LMP are a reactive model in which there are different transition subprobabilities for each action. In this model, uncertainty is (only) considered to be probabilistic; therefore, LMP can be regarded as generalization of deterministic processes. Intimately related to LMP are stochastic relations (Doberkat 2007b); LMP are exactly the stochastic Kripke frames. It will be convenient, in search for motivation, to draw a panorama of the problems addressed by the present work in the context of LMP.

There are several ways to face the problem of 'equivalence of behaviour' or bisimilarity for LMP. One possible but rather arbitrary way to classify them is as relational, coalgebraic, and logical, and at the core of the matter that concerns us are the connections between these different roads. Each one has its own advantages and sharp edges.

\footnotetext{
$\dagger$ Partially supported by CONICET, ANPCyT project PICT 2012-1823, SeCyT-UNC project 05/B284, and EU 7FP grant agreement 295261 (MEALS). Part of this work was presented at Dagstuhl Seminar 12411 on Coalgebraic Logics.
} 
In the relational way, one uses some natural generalizations to continuous spaces of probabilistic bisimilarity as defined by Larsen and Skou (1991). Hence, bisimulations and the relation of bisimilarity are simply binary relations on the base space. This way to define bisimulations is the standard one for Kripke models and will play a central role in this paper. Another, perhaps more important observation is that this relational view is internal: it only works inside a single process. When one wants to compare states in two different processes relationally, it's necessary to construct their direct sum first. Since bisimulations in this sense have no structure (they're plain sets of pairs) facts such as the transitivity of the relation of bisimilarity are straightforward.

In the coalgebraic view, (Doberkat 2009; Rutten 2000) there are two options for describing behaviour of processes: coalgebraic bisimilarity is the existence of a span of zigzag morphisms and the concept of behavioural equivalence, which is given by a cospan of morphisms. Here, the problem of transitivity is not trivial. It can be shown by functorial manipulations that behavioural equivalence is transitive. But in the case of spans, this is a deep theorem. There are two approaches to this problem. One is to apply a technical lemma by Edalat (1999) which ensures the existence of semi-pullbacks in the category of LMP over analytic spaces; that is, every cospan can be completed to a commutative square. The second one allows the construction to be carried out without leaving the realm of Polish spaces, in case one starts with a cospan of LMP over Polish spaces. This was achieved by Doberkat (2005) by using a selection argument. Coalgebraic bisimilarity is a refinement of the relational view, since the mediating process (the 'vertex' of the span) can be regarded as binary relation carrying a LMP structure. Note, however, that the passage to the relational view is not completely smooth, since (Sánchez Terraf 2011, Example 1) shows that direct sum is not always compatible with coalgebraic bisimilarity.

Finally, in the logical view, one fixes a variant of Hennessy-Milner modal logic. Formulas of the modal logic can be regarded as tests on a process and we call two states logically equivalent if they satisfy exactly the same formulas. We don't put logical equivalence under the 'relational approach' since the main ingredients are the logical formulas; moreover, logic is external. There is an alternative but equivalent view, called 'event bisimulation' (Danos et al. 2006) where emphasis is put on families of measurable subsets of the state space. The connection between these two is that the semantics (or validity sets) of Hennessy-Milner formulas are measurable sets for each interpretation (process). This is a recurrent theme that will be exploited in our results. There is a generalization of this logical/event approach through the investigation of the lifting of countably generated equivalence relations to the space of probabilities (Doberkat 2007a).

As in older and related areas as Kripke frames and models, one of the main problems about LMP is to make a link between the logical and the other approaches, specially to relational and coalgebraic bisimilarity. For the former, the problem of logical characterization of bisimilarity on a class of processes consist in proving that (relational) bisimilarity is the same as logical equivalence for each process in the class. One of the cleanest way to do this is to apply the unique structure theorem for analytic spaces, as it was done in the work of Danos et al. (2006). This works for the class of LMP with analytic state spaces, but it can be shown that both notions actually differ in the class 
of LMP over general measurable spaces, and that coalgebraic bisimilarity is not even transitive (Sánchez Terraf 2011) (note that, trivially, logical equivalence is).

Our interest lies in models that include both probability and internal non-determinism. These arise naturally, e.g. by abstraction or underspecification of LMP. In the discrete case, the class of probabilistic automata is an example. Over uncountable state spaces, the common generalization of LMP and probabilistic automata is given by non-deterministic labelled Markov processes (NLMP) (D'Argenio et al. 2009; Wolovick 2012). NLMP allow, for each state $s$ and each action $a$, a (possibly infinite) set of probabilistic behaviours $\mathrm{T}_{a}(s)$. Deterministic NLMP (i.e. for which $\mathrm{T}_{a}(s)$ is a singleton for each $a, s$ ) are essentially the same as LMP. (It's worth to mention a very different approach to underspecification, using super-additive functions, proposed in Desharnais et al. (2011).)

In D'Argenio et al. (2009, 2012), the problem of defining appropriate notions of bisimulation and finding logical characterizations for bisimulation of NLMP was addressed. It turns out that there are three different notions of bisimilarity for these processes: traditional, state-based, and event-based. The first two are 'relational' in nature, and for deterministic NLMP they collapse to state bisimilarity. (It should be noted that a neat coalgebraic presentation of NLMP is still missing, so we won't be concerned with the coalgebraic approach in this work.) Event bisimilarity is analogous to the concept for LMP bearing the same name and hence it is characterized by a logic, though one far more complex than Hennessy-Milner's. It has two levels and uncountably many formulas in the general case.

Indeed, we witness a similar phenomenon as in LTS vs. modal logic, where some logical operators (e.g. conjunction) must have arity at least as the branching of the process. In our case, we can do by just using countable conjunctions, in spite the sets $\mathrm{T}_{a}(s)$ may have the cardinality of the continuum. In any case, by using infinitary operators one is lead to an uncountable logic.

Nevertheless, the arguments used in Danos et al. (2006) can be generalized to encompass image-finite NLMP (i.e. having all the sets $\mathrm{T}_{a}(s)$ finite) over analytic state spaces. Actually, a proof strategy can be found in Celayes (2006); D'Argenio et al. (2009): every countable 'measurable' logic $\mathscr{L}$ satisfying certain local restrictions must characterize bisimilarity. (Here, we call a logic measurable if the validity set of each formula is a measurable subset of the state space.) Both countability and measurability requirements are necessary for the proof to work. As far as we know, all approaches to the problem of logical characterization of bisimilarity need a logic satisfying these two properties.

Several examples in D'Argenio et al. $(2009,2012)$ show that in the case of uncountable branching, our logic does not characterize neither of the relational bisimilarities considered, and that they are different. So the remaining case is the one of countably infinite branching. In this paper, we show that the relation of bisimilarity is not Borel in an appropriate countably branching process $\mathbf{F}$ having a Polish state space, and therefore we prove that there is no countable measurable logic that characterizes bisimilarity in any class of NLMP containing $\mathbf{F}$, thus banishing hope for using the current techniques for proving logical characterization. We will do this in two steps: first we'll see that the relation of bisimilarity on the space $\operatorname{Tr}_{\mathbb{N}}$ of all trees on $\mathbb{N}$ is a non-Borel set (actually, it is analytic-complete). We will then use $\operatorname{Tr}_{\mathbb{N}}$ to build the state space of $\mathbf{F}$, providing a NLMP structure. 
In the next section, we review some of the known results on NLMP, describing the available notions of bisimulation. Most calculations in the paper will be carried on a NLMP where the sets $\mathrm{T}_{a}(s)$ consist entirely of point masses (Dirac's deltas). This simpler model has another, more practical, presentation which is essentially a LTS over a measurable space (Section 2.2). In Section 3, we use some machinery of sequence spaces and unwinding of LTS to assess the complexity of the relation of bisimilarity. The final section contains some concluding remarks.

\section{Review of NLMP}

\subsection{Basic definitions}

All of the material of this section appears in D'Argenio et al. (2012). Let $(S, \Sigma)$ be a measurable space. The set $\Delta(S)$ of probability measures on $(S, \Sigma)$ has a natural $\sigma$-algebra $\Delta(\Sigma)=\sigma\left(\left\{\Delta^{\geqslant q}(Q): q \in \mathbb{Q}, Q \in \Sigma\right\}\right)$, where $\Delta^{\geqslant q}(Q)=\{\mu \in \Delta(S): \mu(Q) \geqslant q\}$. This is the least $\sigma$-algebra making evaluation $\mu \mapsto \mu(Q)$ measurable.

Recall that a Markov kernel on $(S, \Sigma)$ is a measurable map $\mathrm{T}:(S, \Sigma) \rightarrow(\Delta(S), \Delta(\Sigma))$. The following definitions generalize this concept by enlarging the codomain to the family of all measurable sets of probability measures $(\Delta(\Sigma))$, and constructing a $\sigma$-algebra for this family in order to be able to say that $\mathrm{T}$ is measurable. In this section, the $\Lambda$ appearing in Definition 1 will be $\Delta(\Sigma)$; in Section 2.2, the choice of $\Lambda$ will be different.

Definition 1. Let $\Lambda$ be some family of sets. $H(\Lambda)$ is the least $\sigma$-algebra on $\Lambda$ containing all sets $H_{\xi} \doteq\{\zeta \in \Lambda: \zeta \cap \xi \neq \varnothing\}$ with $\xi \in \Lambda$.

Definition 2. A NLMP is a tuple $\mathbf{S}=\left(S, \Sigma,\left\{\mathrm{T}_{a}: a \in L\right\}\right)$, where $\Sigma$ is a $\sigma$-algebra on the set of states $S$, and for each label $a \in L, \mathrm{~T}_{a}:(S, \Sigma) \rightarrow(\Delta(\Sigma), H(\Delta(\Sigma)))$ is measurable. We call $\mathbf{S}$ image-finite (image-countable) if all the sets $\mathrm{T}_{a}(s)$ are finite (countable). NLMP that are not image-finite (-countable) are called image-infinite (-uncountable).

The motivation for the previous definitions is that we want the event 'there exists a probabilistic behavior from $s$ such that ...' to be measurable; we want this in order to be able to calculate the probability of such event (cf. the semantics of the logic below).

Some notation concerning binary relations will be needed to define bisimulations. Let $R$ a binary relation over $S$. A set $Q$ is $R$-closed if $Q \ni x R y$ implies $y \in Q . \Sigma(R)$ is the $\sigma$-algebra of $R$-closed sets in $\Sigma$. If $\mu, \mu^{\prime}$ are measures defined on $\Sigma$, we write $\mu R \mu^{\prime}$ if they coincide on $\Sigma(R)$. Last, let $\Xi$ be a subset of $\operatorname{Pow}(S)$, the powerset of $S$. The relation $\mathcal{R}(\Xi)$ is given by

$$
(s, t) \in \mathcal{R}(\Xi) \quad \Longleftrightarrow \quad \forall Q \in \Xi: s \in Q \Leftrightarrow t \in Q .
$$

\section{Definition 3.}

1. An event bisimulation on a $\operatorname{NLMP}\left(S, \Sigma,\left\{\mathrm{T}_{a}: a \in L\right\}\right)$ is a sub- $\sigma$-algebra $\Lambda$ of $\Sigma$ such that $\mathrm{T}_{a}:(S, \Lambda) \rightarrow(\Delta(\Sigma), H(\Delta(\Lambda)))$ is measurable for each $a \in L$. We also say that a relation $R$ is an event bisimulation if there is an event bisimulation $\Xi$ such that $R=\mathcal{R}(\Xi)$. 
2. A relation $R \subseteq S \times S$ is a state bisimulation if it is symmetric and for all $a \in L, s R t$ implies $\forall \xi \in \Delta(\Sigma(R)): \mathrm{T}_{a}(s) \cap \xi \neq \varnothing \Longleftrightarrow \mathrm{T}_{a}(t) \cap \xi \neq \varnothing$.

3. A relation $R$ is a traditional bisimulation if it is symmetric and for all $a \in L, s R t$ implies that for all $\mu \in \mathrm{T}_{a}(s)$ there exists $\mu^{\prime} \in \mathrm{T}_{a}(t)$ such that $\mu R \mu^{\prime}$.

We say that $s, t \in S$ are traditionally (respective state-, event-) bisimilar, denoted by $s \sim_{\mathrm{t}} t$ $\left(s \sim_{\mathrm{s}} t, s \sim_{\mathrm{e}} t\right)$, if there is a traditional (state, event) bisimulation $R$ such that $s R t$.

We want to stress the fact that each notion of bisimulation/bisimilarity is defined relative to a particular NLMP. Event bisimulation is a straightforward generalization of the same concept for LMP, and it is the one that is most 'compatible' with the measurable structure of the base space. For LMP, it is equivalent to the existence of a cospan of morphisms; see Danos et al. (2006). Traditional bisimulation is in a sense the most faithful generalization of both probabilistic bisimulation by Larsen and Skou and the standard notion of bisimulation for non-deterministic processes, e.g. LTS. Finally, state bisimilarity is a good trade-off between the other two, since it is generally finer than event bisimilarity but it is a little more respectful to the measurable structure than the traditional version; in this case, we only ask transition sets to hit the same $R$-closed sets of measures, and this is a weaker requirement than that of traditional bisimulations. We refer the reader to D'Argenio et al. (2012) for further discussion on these notions of bisimilarity. ${ }^{\dagger}$

To close this section, we introduce the logic $\mathscr{L}$. Consider two kinds of formulas: one that is interpreted on states, and another that is interpreted on measures.

$$
\begin{aligned}
& \varphi \equiv \top\left|\varphi_{1} \wedge \varphi_{2}\right|\langle a\rangle \psi \\
& \psi \equiv \bigvee_{i \in I} \psi_{i}|\neg \psi|[\varphi]_{\geqslant q},
\end{aligned}
$$

where $a \in L, I$ is a countable index set, and $q \in \mathbb{Q} \cap[0,1]$. We denote by $\mathscr{L}$ the set of all formulas generated by the first production. The semantics of the logic is given relative to a $\operatorname{NLMP}\left(S, \Sigma,\left\{\mathrm{T}_{a}: a \in L\right\}\right)$.

$$
\begin{array}{ll}
\llbracket \top \rrbracket=S & \llbracket \bigvee_{i \in I} \psi_{i} \rrbracket=\bigcup_{i} \llbracket \psi_{i} \rrbracket \\
\llbracket \varphi_{1} \wedge \varphi_{2} \rrbracket=\llbracket \varphi_{1} \rrbracket \cap \llbracket \varphi_{2} \rrbracket & \llbracket \neg \psi \rrbracket=\Delta(S) \backslash \llbracket \psi \rrbracket \\
\llbracket\langle a\rangle \psi \rrbracket=\mathrm{T}_{a}^{-1}\left(H_{\llbracket \psi \rrbracket}\right) & \llbracket[\varphi]_{\geqslant q} \rrbracket=\Delta^{\geqslant q}(\llbracket \varphi \rrbracket),
\end{array}
$$

where $\llbracket \chi \rrbracket$ denotes the validity set (or extension) of $\chi$. We may explain a little bit the last two clauses. Expanding the one with the modality, we obtain

$$
\llbracket\langle a\rangle \psi \rrbracket=\left\{s \in S: \exists\left(\mu \in \mathrm{T}_{a}(s) \cap \llbracket \psi \rrbracket\right)\right\},
$$

so that this is the set of all states such that under action $a$, there is a transition to a measure $\mu$ satisfying $\psi$. The test on measures expands to

$$
\llbracket[\varphi]_{\geqslant q} \rrbracket=\{\mu \in \Delta(S): \mu(\llbracket \varphi \rrbracket) \geqslant q\},
$$

so a measure $\mu$ satisfies $[\varphi]_{\geqslant q}$ if it assigns probability greater or equal than $q$ to the set of states satisfying $\varphi$.

$\dagger$ In later works, different (and we hope better) names for these notions are used: state and hit bisimulations, instead of traditional and state bisimulations, respectively. 
Example 4. In case the NLMP $\mathbf{S}=\left(S, \Sigma,\left\{\mathrm{T}_{a}: a \in L\right\}\right)$ is deterministic, meaning $\mathrm{T}_{a}(s)=$ $\left\{k_{a}(s)\right\}$ is a singleton for each $a$ and $s$, it can be proved that $k_{a}$ are Markov kernels and then $\mathbf{S}$ is essentially a LMP. We may easily codify the logic used for these processes (Desharnais 1999) in $\mathscr{L}$ as syntactic sugar; we should only worry about the modality $\langle a\rangle_{q}$ :

$$
\llbracket\langle a\rangle_{q} \varphi \rrbracket=\left\{s \in S: k_{a}(s)(\llbracket \varphi \rrbracket) \geqslant q\right\}=\llbracket\langle a\rangle[\varphi]_{\geqslant q} \rrbracket .
$$

It can be proved by induction that all the sets $\llbracket \chi \rrbracket$ are measurable in the respective spaces by using $H(\Delta(\Sigma))$-measurability. Let $\llbracket \mathscr{L} \rrbracket \doteq\{\llbracket \varphi \rrbracket: \varphi \in \mathscr{L}\}$.

Theorem 5. The logic $\mathscr{L}$ completely characterizes event bisimulation. In other words, $\mathcal{R}(\llbracket \mathscr{L} \rrbracket)=\sim_{\text {e }}$

Theorem 6. $\sim_{\mathrm{t}} \subseteq \sim_{\mathrm{s}} \subseteq \sim_{\mathrm{e}}=\mathcal{R}(\llbracket \mathscr{L} \rrbracket)$.

The following Lemma summarizes one strategy to prove completeness of a logic for traditional bisimilarity. We begin by recalling some definitions; the reader may review them in Kechris (1994) or Srivastava (2008). A topological space $(S, \mathcal{T})$ is Polish if it is separable and completely metrizable and an analytic space is the image of a continuous map between Polish spaces. We call $\mathcal{B}(\mathcal{T}) \doteq \sigma(\mathcal{T})($ or $\mathcal{B}(S)$ if the topology is understood from the context) the Borel $\sigma$-algebra of the space $(S, \mathcal{T})$. We say that a measurable space is an analytic Borel space if it is isomorphic to some $(S, \mathcal{B}(\mathcal{T}))$ with $(S, \mathcal{T})$ analytic.

Lemma 7 (D'Argenio et al. 2009). Let $(S, \Sigma, T)$ be a NLMP with $(S, \Sigma)$ being an analytic Borel space. Let $\mathfrak{L}$ be a logic such that (i) $\mathfrak{L}$ contains operators $\top$ and $\wedge$ with the usual semantics; (ii) for every formula $\varphi \in \mathfrak{L}, \llbracket \varphi \rrbracket \in \Sigma$; (iii) the set of all formulas in $\mathfrak{L}$ is countable; and (iv) for every $s \mathcal{R}(\mathfrak{L}) t$ and every $\mu \in \mathrm{T}_{a}(s)$ there exists $\mu^{\prime} \in \mathrm{T}_{a}(t)$ such that $\forall \varphi \in$ $\mathfrak{L}, \mu(\llbracket \varphi \rrbracket)=\mu^{\prime}(\llbracket \varphi \rrbracket)$. Then, two logically equivalent states $s, t$ are traditionally bisimilar.

The proof of this lemma is based on the unique structure theorem for analytic Borel spaces $(S, \Sigma)$ : every countably generated sub- $\sigma$-algebra of $\Sigma$ that separates points must already be $\Sigma$.

By using this Lemma, we were able to prove that a countable fragment of $\mathscr{L}$ is complete for traditional bisimilarity over image-finite NLMP on analytic state-spaces. The next step would be to prove a similar result for image-countable processes, and the safest way to test this is in a more 'discrete' setting. In the next section, we consider a restricted class of processes.

\subsection{Measurable LTS}

Many interesting (counter)examples can be constructed by considering non-probabilistic NLMP, i.e. one $\mathbf{S}=\left(S, \Sigma,\left\{\mathrm{T}_{a}: a \in L\right\}\right)$ such that for all $a \in L$ and $s \in S, \mathrm{~T}_{a}(s)$ consists entirely of point-masses (i.e. Dirac measures). Assume we have such an $\mathbf{S}$; we may write each set $\mathrm{T}_{a}(s)$ as $\left\{\delta_{x}: x \in \tilde{\mathrm{T}}_{a}(s)\right\}$, where $\tilde{\mathrm{T}}_{a}(s) \subseteq S$ for each $s$ (moreover, it can be seen that $\tilde{\mathrm{T}}_{a}(s) \in \Sigma$ ). Since there is a natural correspondence between points $s \in S$ and Dirac measures $\delta_{s} \in \Delta(S)$, we may discard all references to $\Delta(S)$ and work with the simpler 
structure $\left(S, \Sigma,\left\{\tilde{\mathrm{T}}_{a}: a \in L\right\}\right)$ which is essentially an LTS (with some restrictions) over a measurable space. This presentation of non-probabilistic NLMP appears in Wolovick (2012).

Definition 8. A measurable labelled transition system (MLTS) is a tuple $\mathbf{S}=\left(S, \Sigma,\left\{\tilde{\mathbf{T}}_{a}: a \in\right.\right.$ $L\})$ such that $(S, \Sigma)$ is a measurable space and for each label $a \in L, \tilde{\mathrm{T}}_{a}:(S, \Sigma) \rightarrow(\Sigma, H(\Sigma))$ is a measurable map.

If we write $\langle a\rangle Q$ for $\left\{s: \tilde{\mathrm{T}}_{a}(s) \cap Q \neq \varnothing\right\}$, then the measurability requirement on $\tilde{\mathrm{T}}_{a}$ in Definition 8 amounts asking $\Sigma$ to be stable under the map $\langle a\rangle$ : for all $Q \in \Sigma$, $\langle a\rangle Q \in \Sigma$.

Among the trivial examples of MLTS, we might take any $\operatorname{LTS}\left(S,\left\{\tilde{\mathrm{T}}_{a}: a \in L\right\}\right)$ (where $\tilde{\mathrm{T}}_{a}: S \rightarrow \operatorname{Pow}(S)$ ) and attach to it the powerset $\sigma$-algebra; then the measurability requirements are immediately satisfied. A more interesting one is the following.

Example 9. There is a well-known duality for $\mathrm{BAO}_{\tau}$, the variety of Boolean algebras with operators of type $\tau$ (Blackburn et al. 2006, p. 354ff). Consider the case where the operators $\left\{\nabla_{a}: a \in L\right\}$ in $\tau$ are all unary. Then, the dual category consists of Kripke frames $\left(S,\left\{R_{a}: a \in L\right\}\right)$, where $\nabla_{a}=\langle a\rangle$ for each $a$, endowed with a Stone topology $\mathcal{T}$ such that $\nabla_{a}$ maps clopen sets into clopen sets and the relations $R_{a}$ are point-closed, meaning that $R_{a}[s] \doteq\left\{y \in S: s R_{a} y\right\}$ is closed for each $s \in S$ (see Kracht (1999, Section 4.6)). For such a frame, we can define an associated LTS $\left(S,\left\{\tilde{\mathrm{T}}_{a}: a \in L\right\}\right)$, where $\tilde{\mathrm{T}}_{a}(s) \doteq R_{a}[s]$, and if we augment it with the Borel $\sigma$-algebra, the previous restrictions ensure that $\left(S, \mathcal{B}(\mathcal{T}),\left\{\tilde{\mathrm{T}}_{a}: a \in L\right\}\right)$ is a MLTS.

We have the following correspondence between non-probabilistic NLMP and MLTS.

Proposition 10 (Wolovick 2012, Proposition 4.7). Assume that $\Sigma$ is countably generated and separates points on $S$, and for all $a \in L$ and $s \in S, \mathrm{~T}_{a}(s)=\left\{\delta_{x}: x \in \tilde{\mathrm{T}}_{a}(s)\right\}$ for some sets $\tilde{\mathrm{T}}_{a}(s) \subseteq S$. Then, $\left(S, \Sigma,\left\{\mathrm{T}_{a}: a \in L\right\}\right)$ is a $\operatorname{NLMP}$ iff $\left(S, \Sigma,\left\{\tilde{\mathrm{T}}_{a}: a \in L\right\}\right)$ is a MLTS.

The hypothesis on the measurable structure is satisfied, for example, in any analytic Borel space $(S, \Sigma)$, and in the following sections we will work only with such spaces.

The following Lemma from D'Argenio et al. (2012) provides translations for our three notions of bisimulation. Event bisimulation is again a restatement of the measurability criterion (now that of Definition 8 ) for sub- $\sigma$-algebras, and the reader might notice that for MLTS the notion of traditional bisimulation has a look closer to the standard definitions, e.g. as in Kripke frames.

Lemma 11. Let $\mathbf{S}$ be an NLMP such that for all $a \in L$ and $s \in S, \mathrm{~T}_{a}(s)=\left\{\delta_{x}: x \in \tilde{\mathrm{T}}_{a}(s)\right\}$.

1. A $\sigma$-algebra $\Lambda \subseteq \Sigma$ is an event bisimulation on $\mathbf{S}$ if and only if it is stable under the mapping $\langle a\rangle$.

2. A symmetric relation $R$ is a state bisimulation on $\mathbf{S}$ if and only if for all $s, t \in S$ such that $s R t$, it holds that for all $Q \in \Sigma(R), s \in\langle a\rangle Q \Leftrightarrow t \in\langle a\rangle Q$. 
3. A symmetric relation $R$ is a traditional bisimulation on $\mathbf{S}$ if and only if for all $s, t \in S$ and $u \in \tilde{\mathrm{T}}_{a}(s)$, if $s R t$ then there exists $v \in \tilde{\mathrm{T}}_{a}(t)$ such that $u \mathcal{R}(\Sigma(R)) v$.

Since for every relation one has $R \subseteq \mathcal{R}(\Sigma(R))$, standard (Milner's) bisimilarity $\sim_{\mathrm{M}}$ for LTS is also a traditional bisimulation of MLTS, and then $\sim_{M} \subseteq \sim_{t}$. Traditional bisimilarity for MLTS is coarser than standard bisimilarity because bisimilarity classes are not directly 'accessible,' but only through the measurable subsets. Also, examples in D'Argenio et al. $(2009,2012)$ show that there are (non-probabilistic) image-uncountable NLMP where the notions above are different.

Observe that for a non-probabilistic NLMP as above, the quantitative assessment $q$ in formulas of the type $[\varphi]_{\geqslant q}$ doesn't play any role, and hence $\mathscr{L}$ is equivalent to Hennessy-Milner logic with countable conjunctions (and disjunctions) on this family of processes (for instance, the Hennessy-Milner formula $\langle a\rangle \varphi$ is equivalent to the $\mathscr{L}$-formula $\left.\langle a\rangle[\varphi]_{\geqslant 1}\right)$. Therefore, $\mathscr{L}$ characterizes standard bisimilarity for image-countable MLTS and then $\mathcal{R}(\llbracket \mathscr{L} \rrbracket)=\sim_{\mathrm{M}}$. By appealing to Theorem 6 , we can state

Proposition 12. For image-countable MLTS, all kinds of bisimilarities (traditional, state, event, and standard) coincide.

We shall henceforth drop the subindexes and use simply $\sim$.

Let's return to the problem left open at the end of the previous section. To apply Lemma 7, the candidate logic should satisfy several requirements. So the first question is if there actually exists any countable logic that characterizes bisimilarity for countable LTS. The answer is given by the following:

Example 13 (X. Caicedo). Fix a countable set of labels $L$. There are at most $2^{\aleph_{0}}$ (bisimilarity classes of) countable LTS over $\mathbb{N}$. Hence, there is an injective function $f$ from bisimilarity classes to $\operatorname{Pow}(\mathbb{N})$. Our 'logic' will consist of countably many atomic formulas $P_{n}(n \in \mathbb{N})$ with the following semantics:

$$
\mathbf{S}, s \models P_{n} \Longleftrightarrow n \in f\left([(\mathbf{S}, s)]_{\sim}\right),
$$

where $[\cdot] \sim$ denotes $\sim$-classes of equivalence. The logic $\mathscr{L}_{X}:=\left\{P_{n}: n \in \mathbb{N}\right\}$ is sound and complete for bisimilarity.

The logic $\mathscr{L}_{X}$ is devised in a non-constructive manner, and the main result in this work is to show that actually the extensions of formulas of such a countable logic cannot be Borel sets, confirming the intuition that formulas in $\mathscr{L}_{X}$ cannot be conceived as any reasonable kind of 'test' on a process, no matter how the function $f$ is chosen.

\section{The main result}

We will use some concepts from sequence (zero-dimensional) spaces. The reader may review these and related concepts in Kechris (1994) or Moschovakis (2009). Let $E$ be a set. The set of all finite sequences of elements of $E$ will be denoted by $E^{*}$. The empty sequence will be denoted by $\epsilon$. The $i$ th element of a sequence $s \in E^{*}$ will be denoted by $s^{i}$; hence $s=\left\langle s^{0}, \ldots, s^{|s|-1}\right\rangle$, where $|s|$ is the length of $s$. The concatenation of two sequences 
$s, t \in E^{*}$ will be denoted by $s^{\sim} t$; in case $t=\langle e\rangle$ with $e \in E$, we will write $s^{\sim} e$ instead of $s^{-}\langle e\rangle$. A tree on $E$ is a subset of $E^{*}$ closed by taking prefixes. We will be interested in the case where $E$ is countable, and specially $E=\mathbb{N}$.

Let $A$ be countable, and consider the discrete topology on it. The product space $A^{\mathbb{N}}$ of all infinite sequences of elements of $A$ is Polish and has a (clopen) basis given by the sets $C_{f}=\left\{x \in A^{\mathbb{N}}: f \subset x\right\}$, where $f$ is a finite function. When $A=2$, we obtain the Cantor space $\operatorname{Pow}(\mathbb{N})$. In general, for every countable set $B$, we regard $\operatorname{Pow}(B)$ as a separable compact Hausdorff space where its topology has the following subbasic sets:

$$
\{X \subseteq B: s \in X\},\{X \subseteq B: t \notin X\} \quad \text { with } s, t \in B .
$$

Hence, the basic open sets are $\{X \subseteq B: P \subseteq X \& N \cap X=\varnothing\}$, where $P, N \subseteq B$ are finite; it is also immediate that the Borel $\sigma$-algebra of $\operatorname{Pow}(B)$ is generated by sets of the first kind in Equation (1). In case $B=\mathbb{N}$, we obtain exactly the basis given by $\left\{C_{f}\right\}$. We will use the homeomorphic spaces $2^{B}$ and $\operatorname{Pow}(B)$ (and their respective presentations) interchangeably.

A binary structure $(\mathbb{N}, R)$ can be represented by a point in $\operatorname{Rel} \doteq 2^{\mathbb{N} \times \mathbb{N}}$. The set $L O$ of strict linear order relations,

$$
L O \doteq\left\{R \in 2^{\mathbb{N} \times \mathbb{N}}: R \text { is irreflexive, transitive, and total: } \forall x \neq y, x R \text { or } y R x\right\},
$$

is a closed subset of Rel (Kechris 1994, Section 16.C and 27.C); this can be easily seen since universal conditions (as, for instance, ' $\forall x: x \not R x$ ') define closed sets. Hence, $L O$ is a Polish space. The same happens to the set $\operatorname{Tr}_{\mathbb{N}}$ of all trees on $\mathbb{N}$ : it is a closed subset of $2^{\mathbb{N}^{*}}$.

Let $X$ be a Polish space; recall we are using $\mathcal{B}(X)$ for its Borel sets. $\Sigma_{1}^{1}(X)$ denotes the family

$$
\{A \subseteq X: \exists Y \text { Polish and } f: Y \rightarrow X \text { continuous with } f[Y]=A\}
$$

of analytic subsets of $X$ (see Kechris 1994). Both Borel and analytic sets are preserved by taking continuous pre-images and countable unions and intersections. We will usually omit the reference to the space $X$.

\subsection{Bisimilarity on denumerable trees}

We first recall a standard construction of trees from linear orders. Given a strict linear order $\mathbf{E}=(E, R)$ over a countable set $E$ we may define a new countable structure $\left(T_{\mathbf{E}}, \prec\right)$ as follows:

$-T_{\mathbf{E}} \doteq\left\{s \in E^{*}: s^{|s|-1} R s^{|s|-2} R \ldots R s^{0}\right\} \cup\{\langle e\rangle: e \in E\} \cup\{\epsilon\}$.

$-s \prec s^{\prime} \Longleftrightarrow \exists e \in E: s^{-} e=s^{\prime}$.

The tree $T_{\mathbf{E}}$ consists of all finite decreasing sequences in $(E, R)$. We also use $T_{R}$ to denote this tree, whenever $E$ is clear from the context. We can obviously regard the binary structures $\left(T_{\mathbf{E}}, \prec\right)$ as a LTS with a singleton label set $L \doteq\{l\}$, and we say that two trees are bisimilar if there is a bisimulation relating both of the roots. Our next landmark will be to prove that the relation of bisimilarity among the processes $T_{\mathbf{E}}$ is a non-Borel relation on $\operatorname{Tr}_{\mathbb{N}}$. 
We'll now sketch the argument of the proof. Let $W O(N W O)$ be the set of (non) well-order relations on $\mathbb{N}$, regarded as subsets of $L O$. It is well known that the set $N W O$ is a $\boldsymbol{\Sigma}_{1}^{1}$-complete set, in the sense that it is as 'complicated' as any analytic subset of a (zero-dimensional) Polish space; in particular, it is not Borel. We will be able to distinguish elements $R \in N W O$ among linear orderings essentially just by looking at the bisimilarity type of the tree $T_{R}$ over $\mathbb{N}$, regarded as a processes with initial state $\epsilon$. Thus, we'll have succeeded in reducing NWO to the relation of bisimilarity, thus showing that the latter is not Borel.

In first place, we elucidate the notion of reduction that concerns us.

Definition 14. Let $X, Y$ be Polish spaces and $A \subseteq X$ and $B \subseteq Y$. A continuous reduction of $A$ to $B$ is a continuous map $f: X \rightarrow Y$ such that $f^{-1}[B]=A$; in this case, we say that $A$ is Wadge reducible to $B . B$ is $\Sigma_{1}^{1}$-hard if for every zero-dimensional Polish space $X$ and every $A \in \Sigma_{1}^{1}(X), A$ is Wadge reducible to $B$, and $B$ is $\Sigma_{1}^{1}$-complete if moreover, $B \in \Sigma_{1}^{1}(X)$.

Theorem 15 (Lusin, Sierpiński). NWO is a $\Sigma_{1}^{1}$-complete and, in particular, non-Borel subset of LO (Kechris 1994, 27.12).

There is an extensive literature on $\boldsymbol{\Sigma}_{1}^{1}$-complete sets; one of the prominent examples is the relation of isomorphism on denumerable structures, and in particular for binary structures.

Theorem 16. The relation of isomorphism between denumerable binary structures, coded as a subset of $2^{\mathbb{N} \times \mathbb{N}} \times 2^{\mathbb{N} \times \mathbb{N}}$ is $\Sigma_{1}^{1}$-complete (Kechris 1994 (27.D), 2011). ${ }^{\dagger}$

Looking into the detail of the argument, we argue that the trees $T_{R}$ corresponding to $R \in W O$ are well founded: regarded as processes, they're terminating. The depth of such a tree equals the length of the well-order $R$ and since this depth can be 'measured' by using infinitary modal formulas, non-isomorphic well orders have non-bisimilar trees.

In the case of an order relation $S$ on $\mathbb{N}$ that is not a well order, there must be an infinite branch in $T_{S}$ (and hence it can't be bisimilar to any $T_{R}$ with $R \in W O$ ), but we can say more: the tree $T_{S}$ is bisimilar to the process that results after attaching a loop to the initial state of the tree $T^{\prime}$ associated to the maximal well-ordered initial segment of $(\mathbb{N}, S)$. For the purpose of having a manageable example, consider the linear orders $\mathbf{L} \doteq\left(\{0,1,2\},<_{3}\right)$ and $\mathbf{L}^{\prime} \doteq\left(\{0,1,2\} \cup\{\ldots,-3,-2,-1\},<^{\prime}\right)$, where $a<^{\prime} b$ if $b$ is negative and $a$ is not, and otherwise $<^{\prime}$ behaves as the ordering of the integers. Then, the trees corresponding to each of them are respectively bisimilar to the processes in the following picture:

\footnotetext{
$\dagger$ Another examples of (Borel) $\Sigma_{1}^{1}$-complete relations are those of isomorphism and embedding between separable Banach spaces, and the relation ' $F$ and $F^{\prime}$ have non-empty intersection' in the space of closed subsets of $\mathbb{N}^{\mathbb{N}}$.
} 

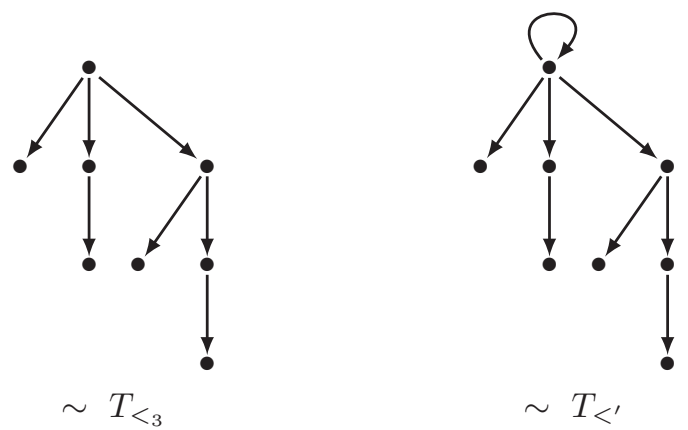

We will show that the bisimilarity type of $T_{S}$ for non-well founded $S$ only depends on the order type of the maximal well-ordered initial segment of $(\mathbb{N}, S)$. Continuing with the previous example, observe that $\mathbf{L}^{\prime}$ is the ordered sum of the poset $\mathbf{L}$ and the poset $\mathbf{M}$ of the negative integers; we denote this ordered sum as $\mathbf{L}+\mathbf{M}$. Although the posets $\mathbf{L}^{\prime}$ and $\mathbf{L}^{\prime}+\mathbf{L}^{\prime}$ are not isomorphic, they have the same maximal well-ordered initial segment (i.e. L) and hence, $T_{\mathbf{L}^{\prime}}$ and $T_{\mathbf{L}^{\prime}+\mathbf{L}^{\prime}}$ are bisimilar.

Now, we may apply the following idea by Dougherty used in one proof (outlined in Kechris 2011) of Theorem 16. If $\mathbf{L}$ is a well order, then $\mathbf{L}+\mathbf{L}$ is itself a well order and $T_{\mathbf{L}} \nsim T_{\mathbf{L}+\mathbf{L}}$ since $\mathbf{L} \neq \mathbf{L}+\mathbf{L}$. But in case $\mathbf{L}$ is not, the trees $T_{\mathbf{L}}$ and $T_{\mathbf{L}+\mathbf{L}}$ are indeed bisimilar since $\mathbf{L}$ and $\mathbf{L}+\mathbf{L}$ have the same maximal well-ordered initial segment.

Then, the reduction we are looking for is given by the map $R \mapsto\left(T_{R}, T_{R+R}\right)$, where $R \in L O$.

We'll begin by proving that this map is indeed continuous.

Lemma 17. The map $R \mapsto T_{R}$ from $L O$ to $2^{\mathbb{N}^{*}}$ is continuous.

Proof. We will show that the function from $2^{\mathbb{N} \times \mathbb{N}}$ to $2^{\mathbb{N}^{*}}$ with the same definition is continuous. It is enough to prove that the pre-image by $T$. of sub-basic elements are open. Assume $s \in \mathbb{N}^{*}$. Then,

$$
R \in(T .)^{-1}\left[\left\{A \subseteq 2^{\mathbb{N}^{*}}: s \in A\right\}\right] \Longleftrightarrow s \in T_{R} \Longleftrightarrow \forall j: 0<j<|s| \Rightarrow s^{j} R s^{j-1} .
$$

Hence, the pre-image can be written in the form

$$
(T .)^{-1}\left[\left\{A \subseteq 2^{\mathbb{N}^{*}}: s \in A\right\}\right]=\left\{R \subseteq \mathbb{N} \times \mathbb{N}:\left\{\left(s^{|s|-1}, s^{|s|-2}\right), \ldots,\left(s^{1}, s^{0}\right)\right\} \subseteq R\right\},
$$

which is basic open. The other case is very similar. For $t \in \mathbb{N}^{*}$,

$$
t \notin T_{R} \Longleftrightarrow \exists j: 0<j<|t| \& t^{j} \not R t^{j-1},
$$

hence, we have

$$
(T .)^{-1}\left[\left\{A \subseteq 2^{\mathbb{N}^{*}}: t \notin A\right\}\right]=\bigcup_{0<j<|t|}\left\{R \subseteq \mathbb{N} \times \mathbb{N}:\left(t^{j}, t^{j-1}\right) \notin R\right\},
$$

again an open set. 
We'll need a definition of sum of linear orders such that $L O$ is closed under this operation. Given $R, R^{\prime} \in L O$, let

$$
\left(R+R^{\prime}\right)(n, m) \doteq \begin{cases}1 & 2 \mid n \& 2 \nmid m \\ R\left(\frac{n}{2}, \frac{m}{2}\right) & 2|n \& 2| m \\ R^{\prime}\left(\frac{n-1}{2}, \frac{m-1}{2}\right) & 2 \nmid n \& 2 \nmid m \\ 0 & 2 \nmid n \& 2 \mid m .\end{cases}
$$

Hence, we have the following straightforward lemma, and its corollary rounds out the proof of continuity.

Lemma 18. $\left(\mathbb{N}, R+R^{\prime}\right)$ is isomorphic to the ordered sum of $(\mathbb{N}, R)$ and $\left(\mathbb{N}, R^{\prime}\right)$, and $\left(R, R^{\prime}\right) \mapsto R+R^{\prime}$ is continuous from $L O \times L O$ to $L O$.

Corollary 19. The map $R \mapsto\left(T_{R}, T_{R+R}\right)$ is continuous from $L O$ to $\operatorname{Tr}_{\mathbb{N}} \times \operatorname{Tr}_{\mathbb{N}}$.

Now, we want to check that well orders of different type can't give rise to bisimilar trees. For that, we define by recursion on $\alpha<\omega_{1}$ the following modal formulas:

$-\varphi_{0} \doteq \top$.

$-\varphi_{\alpha+1} \doteq \diamond \varphi_{\alpha}$.

$-\varphi_{\lambda} \doteq \bigwedge_{\beta<\lambda} \varphi_{\beta}$, for limit $\lambda$.

When satisfied at the root of a tree, formula $\varphi_{\alpha}$ states that its depth is as least $\alpha$. We record two standard results.

Proposition 20. For a well-order $(\mathbb{N}, R)$ of type $\alpha, T_{R}, \epsilon \models \varphi_{\beta}$ if and only if $\beta \leqslant \alpha$. If $(\mathbb{N}, R)$ is not well founded, $T_{R}, \epsilon \models \varphi_{\beta}$ for all $\beta<\omega_{1}$.

Corollary 21. If $(\mathbb{N}, R)$ is a well order and $R^{\prime} \in L O, T_{R}, \epsilon \sim T_{R^{\prime}}, \epsilon \Longleftrightarrow(\mathbb{N}, R) \cong\left(\mathbb{N}, R^{\prime}\right)$. In particular, $T_{R}, \epsilon \nsim T_{R+R}, \epsilon$.

Now, we turn to the proof that if $\mathbf{L}^{\prime}$ is not a well order, then the trees $T_{\mathbf{L}^{\prime}}$ and $T_{\mathbf{L}^{\prime}+\mathbf{L}^{\prime}}$ are bisimilar. For this, we will characterize the process $T_{\mathbf{A}+\mathbf{B}}$ corresponding to the ordered sum of posets $\mathbf{A}$ and $\mathbf{B}$ by using a kind of disrupt construction between $T_{\mathbf{A}}$ and $T_{\mathbf{B}}$. More generally, take two processes (represented by binary structures) $\mathbf{C}=(C, R)$ and $\mathbf{D}=(D, Q)$ and 'execute them in succession': taking $c \in C$ and $d \in D$, we define a new state $c \triangleright d$ that starts behaving as $c$ but in any moment can be 'disrupted' and then it behaves as $d^{\dagger}$. Formally, let two binary structures $\mathbf{C}$ and $\mathbf{D}$ as above be given with $C$ and $D$ disjoint, and define $\mathbf{C} \triangleright \mathbf{D}$ to be a new binary structure with universe $(C \times D) \cup D$ (where we write an ordered pair $(c, d)$ as $c \triangleright d)$ equipped with the least binary relation ' $\rightarrow$ ' such that

$$
\begin{aligned}
c \triangleright d \rightarrow c^{\prime} \triangleright d & \Longleftrightarrow c R c^{\prime} \\
c \triangleright d \rightarrow d^{\prime} & \Longleftrightarrow d Q d^{\prime} \\
d \rightarrow d^{\prime} & \Longleftrightarrow d Q d^{\prime},
\end{aligned}
$$

\footnotetext{
$\dagger$ For the process-algebra minded, the operator $\triangleright$ is very much like LOTOS disabling operator (Bolognesi and Brinksma 1987).
} 
for all $c, c^{\prime} \in C$ and $d, d^{\prime} \in D$. In the following Lemma, the binary structures $\mathbf{C}$ and $\mathbf{D}$ considered are trees with $R$ and $Q$ both being the successor relation $\prec$.

Lemma 22. Let $\mathbf{A}=(A, R)$ and $\mathbf{B}=(B, T)$ be strict linear orders with $A$ and $B$ disjoint. Then, $T_{\mathbf{A}+\mathbf{B}}, \epsilon \sim T_{\mathbf{B}} \triangleright T_{\mathbf{A}}, \epsilon \triangleright \epsilon$.

Proof. Observe that any decreasing sequence in $\mathbf{A}+\mathbf{B}$ decomposes uniquely as the concatenation of a sequence $s$ in $\mathbf{A}$ after a sequence $t$ in $\mathbf{B}$. We'll check that the relation

$$
\theta \doteq\left\{(t, t \triangleright \epsilon): t \in T_{\mathbf{B}}\right\} \cup\left\{\left(t^{\top} s, s\right): t \in T_{\mathbf{B}}, s \in T_{\mathbf{A}} \backslash\{\epsilon\}\right\}
$$

is a bisimulation. Note that $(\epsilon, \epsilon \triangleright \epsilon) \in \theta$.

We check the forth and back property for each of type of pairs in $\theta$. Assume $(t, t \triangleright \epsilon) \in \theta$, hence $t \in T_{\mathbf{B}}$ (i.e. it is a decreasing sequence in $\mathbf{A}+\mathbf{B}$ with no elements from $A$ ). Further, assume we have a 'transition' $t<t^{-} b$ in $T_{\mathbf{A}+\mathbf{B}}$ with $b \in B$ (this means that $t^{-} b \in T_{\mathbf{B}}$ ). The forth property follows immediately since $t \triangleright \epsilon \rightarrow t^{-} b \triangleright \epsilon$ by Equation (2) and $\left(t^{-} b, t^{-} b \triangleright \epsilon\right) \in \theta$. In case of a transition $t \prec t^{-} a$ with $a \in A$, we first see that $t \triangleright \epsilon \rightarrow\langle a\rangle$ by Equation (3) (with $c=t, d=\epsilon$, and $d^{\prime}=\langle a\rangle$ ). But now $\left(t^{-} a,\langle a\rangle\right) \in \theta$, and the forth property follows. The back property is checked by using very similar arguments.

Now assume $\left(t^{-} s, s\right) \in \theta$, where $s \in T_{\mathbf{A}}$ and $t \in T_{\mathbf{B}}$. Then,

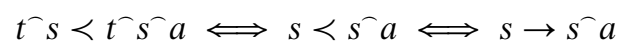

for each $a \in A$, where the last equivalence holds by Equation (4) (with $d=s, d^{\prime}=s^{\wedge} a$ ). This gives us the forth and back property for this case.

Lemma 23. If $\mathbf{A}=(A, R)$ is a countable strict linear order without first element, then $\left(T_{\mathbf{A}}, \prec, \epsilon\right) \cong\left(\mathbb{N}^{*}, \prec, \epsilon\right)$. In particular, $T_{\mathbf{A}}, \epsilon \sim \mathbb{N}^{*}, \epsilon$.

Proof. It is enough to note that under the hypothesis, $T_{\mathbf{A}}$ is a (countable) tree in which every node has infinitely many successors.

Corollary 24. If $R \in N W O, T_{R}, \epsilon \sim T_{R+R}, \epsilon$.

Proof. Decompose $\mathbf{N} \doteq(\mathbb{N}, R)$ as $\mathbf{A}+\mathbf{B}$, where $\mathbf{A}$ is the maximal well-ordered initial segment of $\mathbf{N}$ and hence $\mathbf{B}$ has no first element. Then, $\mathbf{N}+\mathbf{N} \cong \mathbf{A}+\mathbf{B}+\mathbf{A}+\mathbf{B} \cong \mathbf{A}+\mathbf{C}$, where $\mathbf{C} \doteq \mathbf{B}+\mathbf{A}+\mathbf{B}$ has no first element. Hence,

$$
\begin{aligned}
T_{R+R}, \epsilon & \sim T_{\mathbf{A}+\mathbf{C}}, \epsilon & & \text { since } T_{R+R}=T_{\mathbf{N}+\mathbf{N}} \cong T_{\mathbf{A}+\mathbf{C}}, \\
& \sim T_{\mathbf{C}} \triangleright T_{\mathbf{A}}, \epsilon \triangleright \epsilon & & \text { by Lemma } 22, \\
& \sim T_{\mathbf{B}} \triangleright T_{\mathbf{A}}, \epsilon \triangleright \epsilon & & \text { by Lemma } 23, T_{\mathbf{C}} \cong T_{\mathbf{B}} \\
& \sim T_{\mathbf{A}+\mathbf{B}, \epsilon} & & \text { by Lemma } 22, \\
& \sim T_{R}, \epsilon . & &
\end{aligned}
$$

At this point, we can gather all the previous lemmas and prove the following technical result, which is the main ingredient for the developments in the next section.

Theorem 25. Bisimilarity on $\operatorname{Tr}_{\mathbb{N}}$ is $\boldsymbol{\Sigma}_{1}^{1}$-hard, and hence not Borel. 
Proof. We have seen that $R \mapsto f(R) \doteq\left(T_{R}, T_{R+R}\right)$ is continuous by Corollary 19. By Corollary 21 and Corollary 24, $f^{-1}[\sim]=N W O$ and hence $N W O$ is Wadge reducible to $\sim$. This makes $\sim \Sigma_{1}^{1}$-hard, and not Borel (since Borel sets are preserved by continuous pre-images).

\subsection{Bisimilarity on MLTS is not Borel}

In this section, we will obtain our main result. For this, we will construct a MLTS F for which the relation of bisimilarity is not Borel. It is easy to show that for any countable measurable logic and for any MLTS $\mathbf{S}$, the induced relation of logical equivalence on $\mathbf{S}$ is Borel, so the punchline is that there is no such a logic for any class of MLTS containing F.

The state space of $\mathbf{F}$ will be the result of collecting all the tree processes $T_{R}$ (with an appropriate 'tagging'), in such a way that bisimilarity on $\mathbf{F}$ is exactly the relation of bisimilarity on $\operatorname{Tr}_{\mathbb{N}}$. The only details to be taken care of are essentially the measurability requirements to be a MLTS.

Let $L \doteq\{l\}$ be the singleton label set. Define $\mathbf{F}=(F, \mathcal{B}(F), \bar{\prec})$ (where we are taking $\left.\tilde{\mathrm{T}}_{l}=\bar{\prec}\right)$ such that

$-(F, \mathcal{B}(F)) \doteq\left(\operatorname{Tr}_{\mathbb{N}} \times \mathbb{N}^{*}, \mathcal{B}\left(\operatorname{Tr}_{\mathbb{N}} \times \mathbb{N}^{*}\right)\right)\left(\right.$ where $\mathbb{N}^{*}$ is considered discrete $)$. Note that this is a Polish space.

- $(T, s) \doteq\left\{\left(T, s^{\prime}\right): s, s^{\prime} \in T \& s \prec s^{\prime}\right\}$.

We prove that $\mathbf{F}$ is a MLTS. First note that sets $\prec(T, s)$, being countable, are Borel. We will also use the symbol $\prec$ also as a binary relation, defined in the obvious way: $(T, s) \bar{\prec}\left(T^{\prime}, s^{\prime}\right)$ iff $T=T^{\prime}, s, s^{\prime} \in T$ and $s \prec s^{\prime}$.

For a subset $A \subseteq X \times Y$ of a product and $c \in Y$, the section $\left.A\right|_{c}$ is the set $\{x \in X$ : $(x, c) \in A\}$, the pre-image of the injection $x \mapsto(x, c)$.

Lemma 26. $\langle l\rangle Q$ is Borel for each $Q \in \mathcal{B}\left(\operatorname{Tr}_{\mathbb{N}} \times \mathbb{N}^{*}\right)$, and hence $\mathbf{F}$ is a MLTS.

Proof.

$$
\begin{aligned}
& \langle l\rangle Q=\left\{(T, s) \in F: \exists\left(T^{\prime}, s^{\prime}\right) \in Q\left((T, s) \bar{\prec}\left(T^{\prime}, s^{\prime}\right)\right)\right\} \\
& =\left\{(T, s) \in F: \exists s^{\prime}\left(\left(T, s^{\prime}\right) \in Q \&(T, s) \prec\left(T, s^{\prime}\right)\right)\right\} \\
& =\left\{(T, s) \in F: \exists n \in \mathbb{N}\left(s^{\sim} n \in T \&\left(T, s^{-} n\right) \in Q\right)\right\} \\
& =\bigcup_{n \in \mathbb{N}} \bigcup_{s \in \mathbb{N}^{*}}\left\{(T, s) \in F: s^{\curlyvee} n \in T \&\left(T, s^{\curlyvee} n\right) \in Q\right\} \text {. }
\end{aligned}
$$

Now, we may write the set inside the unions (now for fixed $s, n$ ) as

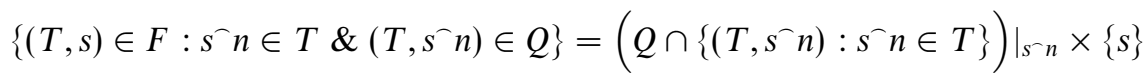

$$
\begin{aligned}
& =\left.\left(Q \cap\left(\left\{T: s^{\sim} n \in T\right\} \times\left\{s^{\sim} n\right\}\right)\right)\right|_{s\urcorner n} \times\{s\} .
\end{aligned}
$$

The inner rectangle is clopen, and since $Q$ is Borel, the set between the big parentheses is Borel. The whole set is easily Borel, too. 
Theorem 27. The relation of bisimilarity is a $\Sigma_{1}^{1}$-hard subset of $F \times F$, and hence not Borel.

Proof. We will reduce again NWO to bisimilarity. By Proposition 12, we can consider standard bisimilarity, since $\mathbf{F}$ is image-countable. It is immediate that states $(T, \epsilon)$ and $\left(T^{\prime}, \epsilon\right)$ are bisimilar in $\mathbf{F}$ if and only if there is a bisimulation between the tree processes $T$ and $T^{\prime}$. Since the injection $T \mapsto(T, \epsilon)$ is continuous from $\operatorname{Tr}_{\mathbb{N}}$ to $\operatorname{Tr}_{\mathbb{N}} \times \mathbb{N}^{*}$, the composition $R \mapsto f(R) \doteq\left(\left(T_{R}, \epsilon\right),\left(T_{R+R}, \epsilon\right)\right)$ also is (by Lemmas 17 and 18). This $f$ is a suitable reduction, since $f^{-1}[\sim]=N W O$.

We arrive at the main result of this work.

Theorem 28. There is no countable logic $\mathscr{L}$ that characterizes bisimulation on $\mathbf{F}$ such that $\llbracket \mathscr{L} \rrbracket \subseteq \mathcal{B}(F)$.

Proof. Assume $\mathcal{R}(\llbracket \mathscr{L} \rrbracket)=\sim$. Then,

$$
s \sim t \Longleftrightarrow(s, t) \in \bigcap\{(\llbracket \varphi \rrbracket \times \llbracket \varphi \rrbracket) \cup((F \backslash \llbracket \varphi \rrbracket) \times(F \backslash \llbracket \varphi \rrbracket)): \varphi \in \mathscr{L}\} .
$$

This contradicts Theorem 27, since the right-hand side is a Borel definition of $\sim$.

\subsection{Bisimilarity is $\boldsymbol{\Sigma}_{1}^{1}$-complete}

We finally show in this section that bisimilarity on $\mathbf{F}$ behaves similarly to the isomorphism relation on countable structures: it is an analytic equivalence relation with Borel classes. Since we already proved it to be $\Sigma_{1}^{1}$-hard, we would have seen it is a complete analytic set.

We will need a technical tool that allows us to obtain a canonical representative of the bisimilarity type of a tree (see Blackburn et al. (2006, p. 275), Corollary 47 and the paragraphs before). That is, for each tree $T$ we obtain a new tree $\boldsymbol{\Omega}_{T}$ such that $T, \epsilon \sim T^{\prime}, \epsilon$ if and only if $\boldsymbol{\Omega}_{T} \cong \boldsymbol{\Omega}_{T^{\prime}}$. We will essentially show that the map $T \mapsto \boldsymbol{\Omega}_{T}$ is continuous, thereby reducing the relation of bisimilarity on $\operatorname{Tr}_{\mathbb{N}}$ (and on $\mathbf{F}$ ) to isomorphism of countable structures.

An $\omega$-indexed path from $s \in S$ on a $\operatorname{LTS} \mathbf{S}=(S, R)$ is a sequence $u$ of the form

$$
u=s_{0}\left(s_{1}, a_{1}\right)\left(s_{2}, a_{2}\right) \ldots\left(s_{n}, a_{n}\right)
$$

such that $s_{0}=s, a_{i} \in \mathbb{N}$ for all $i$, and $\left(s_{i-1}, s_{i}\right) \in R$ for $i=1, \ldots, n$. The $\omega$-expansion at $s$ of a LTS $\mathbf{S}$ is the LTS $\bar{\Omega}_{\mathbf{S}}(s)=(\bar{\Omega}, \bar{R})$ such that $\bar{\Omega}$ is the set of all $\omega$-indexed paths on $\mathbf{S}$ from $s$ and the relation $\bar{R}$ is defined by $(u, v) \in \bar{R}$ iff $v$ has the form $u(s, a)$ for some $a$ and $s$.

Since we are dealing with trees on $\mathbb{N}$, the latter construction provides us with another tree that it is easily seen to be isomorphic to the one given by the following alternative description.

Definition 29. The $\omega$-expansion of $(T, \prec)$ at $s$ is the $\operatorname{LTS} \boldsymbol{\Omega}_{T}(s)=\left(\Omega_{T}(s), \bar{R}_{T}(s)\right)$ such that $\Omega_{T}(s)=\{(t, n): s \subseteq t \in T \& n \in \omega\}$ and the relation $\bar{R}_{T}(s) \subseteq(T \times \mathbb{N})^{2}$ is given by

$$
(u, n) \bar{R}_{T}(s)(t, m) \Longleftrightarrow u \prec t .
$$


The importance of this construction lies in the fact that two states in a countable tree are bisimilar if and only if they have isomorphic $\omega$-expansions. Note that the relation $\bar{R}_{T}(s)$ can be defined uniformly for all $T \in \operatorname{Tr}_{\mathbb{N}}$ and all $s \in T$. For this, we take care of the requirement ' $\bar{R}_{T}(s) \subseteq(T \times \mathbb{N})^{2}$ ' by writing

$$
(u, n) \bar{R}_{T}(s)(t, m) \Longleftrightarrow s \subseteq u \& u \prec t \& t \in T,
$$

since the right-hand side implies $s, u \in T$. Hence, we are considering the function $\bar{R}$ : $\operatorname{Tr}_{\mathbb{N}} \times \mathbb{N}^{*} \rightarrow 2^{\left(\mathbb{N}^{*} \times \mathbb{N}\right)^{2}}$ defined by $\bar{R}(T, s) \doteq \bar{R}_{T}(s)$. Note that $\bar{R}(T, s)=\varnothing$ if $s \notin T$.

Lemma 30. Bisimilarity classes on $\mathbf{F}$ are Borel.

Proof. By the previous observations, we conclude that for each $(T, s) \in F$, the bisimilarity class $[(T, s)]_{\sim}$ is mapped by $\bar{R}$ into the isomorphism class $[\bar{R}(T, s)]_{\cong}$. Then, $[(T, s)]_{\sim}=\bar{R}^{-1}\left([\bar{R}(T, s)]_{\cong}\right)$. By Scott's Theorem (1964), we know that isomorphism classes of countable (binary) structures are Borel, hence $[\bar{R}(T, s)] \cong$ is a Borel subset of $2^{\left(\mathbb{N}^{*} \times \mathbb{N}\right)^{2}}$. Then, we just have to show that the map $\bar{R}$ is Borel measurable. We'll actually see that it is continuous.

It is enough to show that pre-images of sub-basic sets are open. Take $((u, n),(t, m)) \in$ $\left(\mathbb{N}^{*} \times \mathbb{N}\right)^{2}$; we have two cases. If $u<t$,

$$
\begin{aligned}
\bar{R}^{-1}[\{R:((u, n),(t, m)) \in R\}] & =\{(T, s): t \in T \& s \subseteq u\} \\
& =\{T: t \in T\} \times\{s: s \subseteq u\},
\end{aligned}
$$

and it is empty otherwise. The last set is an open rectangle.

Now, we go for the other type of sub-basic open sets. If $u \nless t$,

$$
\bar{R}^{-1}[\{R:((u, n),(t, m)) \notin R\}]=\operatorname{Tr}_{\mathbb{N}} \times \mathbb{N}^{*} .
$$

Otherwise,

$$
\begin{aligned}
\bar{R}^{-1}[\{R:((u, n),(t, m)) \notin R\}] & =\{(T, s): t \notin T \text { or } s \nsubseteq u\} \\
& =\left(\{T: t \notin T\} \times \mathbb{N}^{*}\right) \cup\left(\operatorname{Tr}_{\mathbb{N}} \times\{s: s \nsubseteq u\}\right),
\end{aligned}
$$

again an open set.

By the proof of the previous lemma, $\bar{R}$ is a reduction showing that bisimilarity is $\Sigma_{1}^{1}$, since isomorphism is. We also give a direct proof of this fact, by analyzing an explicit definition of $\sim$ on $\mathbf{F}$. We need an auxiliary calculation first.

Lemma 31. Let $A$ be countable, $Y$ Polish and $B_{k} \subseteq Y$ Borel for all $k \in A$. Then,

$$
C(R, y) \stackrel{\bullet}{\Longleftrightarrow} \forall k \in R:\left(y \in B_{k}\right)
$$

is Borel in $2^{A} \times Y$. 
Proof. We have $(R, y) \in C \Longleftrightarrow \forall k \in A:\left(k \in R \Rightarrow y \in B_{k}\right)$. Then,

$$
\begin{aligned}
C & =\bigcap_{k \in A}\left\{(R, y): k \in R \Rightarrow y \in B_{k}\right\} \\
& =\bigcap_{k \in A}\{(R, y): k \notin R\} \cup\left\{(R, y): y \in B_{k}\right\} \\
& =\bigcap_{k \in A}(\{R: k \notin R\} \times Y) \cup\left(2^{A} \times B_{k}\right)
\end{aligned}
$$

which is obviously Borel.

Theorem 32. Bisimilarity on $\mathbf{F}$ is $\boldsymbol{\Sigma}_{1}^{1}$.

Proof. As usual, $n, m$ denote non-negative integers and $s_{i}$ finite sequences. The definition of bisimilarity on $\mathbf{F}$ is as follows:

$$
\begin{aligned}
& \left(T_{1}, s\right) \sim\left(T_{2}, s^{\prime}\right) \Longleftrightarrow \exists R \in 2^{\mathbb{N}^{*} \times \mathbb{N}^{*}}:\left(s, s^{\prime}\right) \in R \& \\
& \& \forall s_{1} \forall s_{2} \forall n .\left(s_{1}-n \in T_{1} \& s_{2} \in T_{2} \&\left(s_{1}, s_{2}\right) \in R \Longrightarrow\right. \\
& \left.\exists m: s_{2} \uparrow m \in T_{2} \&\left(s_{1} \uparrow n, s_{2} \uparrow m\right) \in R\right) \& \\
& \& \forall s_{1} \forall s_{2} \forall n .\left(s_{1} \in T_{1} \& s_{2} \uparrow n \in T_{2} \&\left(s_{1}, s_{2}\right) \in R \Longrightarrow\right. \\
& \left.\exists m: s_{1} \uparrow m \in T_{1} \&\left(s_{1} \uparrow m, s_{2} \uparrow n\right) \in R\right) .
\end{aligned}
$$

It suffices to prove that the set defined inside the outer existential quantifier is Borel in $2^{\mathbb{N}^{*} \times \mathbb{N}^{*}} \times F \times F$. We first consider the third line of the definition. The set defined by

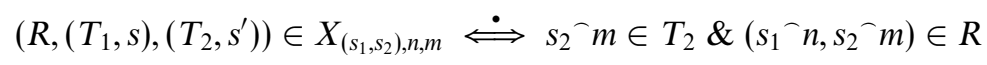

is easily Borel. Then, the condition $\exists m: s_{2}{ }^{-} m \in T_{2} \&\left(s_{1}{ }^{-} n, s_{2}{ }^{-} m\right) \in R$ also is and



finally defines a Borel set of tuples $\left(R,\left(T_{1}, s\right),\left(T_{2}, s^{\prime}\right)\right)$ indexed by elements $\left(s_{1}, s_{2}\right) \in R$. We may apply now Lemma 31 and conclude that

$$
\forall\left(s_{1}, s_{2}\right) \in R: \forall n \cdot\left(s_{1}^{\curlyvee} n \in T_{1} \& s_{2} \in T_{2} \Longrightarrow \exists m: s_{2}\left\lceil m \in T_{2} \&\left(s_{1}^{\curlyvee} n, s_{2} \uparrow m\right) \in R\right),\right.
$$

which is equivalent to the second and third lines of our definition for bisimilarity, is Borel.

The rest of the formula is handled similarly.

By using Theorem 27, we conclude

Corollary 33. Bisimilarity on $\mathbf{F}$ is $\Sigma_{1}^{1}$-complete. 


\section{Conclusion}

NLMP combine probabilistic behaviour with internal non-determinism, over uncountable state spaces. In this framework, we considered the problem of describing bisimilarity by using a modal logic.

We reviewed the different available notions of 'equivalence of behaviour'. They proceed, in some way, from analogous concepts for LMP. So the problem of logical characterization of bisimilarity is manifold, depending on which notion of bisimulation one is concerned. In D'Argenio et al. (2012), it was established that the three concepts of bisimilarity (traditional, state, and event) are indeed different. The counterexamples were imageuncountable process, i.e. having an uncountable number of probabilistic behaviours for each pair 〈state,action〉. For only one of these concepts (event bisimilarity) did the logical characterization go through, in a similar way it was done for LMP in Danos et al. (2006).

In the other extreme of the spectrum, the case of image-finite NLMP was completely solved in D'Argenio et al. (2012), where all bisimilarities coincide and they are characterized by a neat modal logic.

The case left was that of image-denumerable processes. In this restricted setting, we have that traditional and state bisimilarity coincide and the logic characterizing the event based has uncountably many formulas. But in all approaches to logical characterization, a countable measurable logic is needed.

We restricted the image-denumerable case further by considering only non-probabilistic NLMP, and its equivalent formulation as MLTS. With this simplification, we are led essentially to consider plain LTS and Milner's bisimilarity since this is the same as the other notions on image-countable MLTS. We showed that for the latter family of processes, there is no countable measurable logic characterizing bisimilarity. We did this in a very strong sense, by pointing out a specific NLMP F whose base space $F$ is Polish such that bisimilarity on $\mathbf{F}$ is an analytic non-Borel subset of $F^{2}$; therefore there is no countable measurable logic that characterizes bisimilarity for this process, and a fortiori, for any class of processes containing $\mathbf{F}$ (e.g. the class of all image-infinite NLMP with a Polish state-space). As an intermediate technical step, we proved that the relation of bisimilarity on the Polish space of all trees on $\mathbb{N}$ is analytic-complete. Then, the space $F$ is essentially this Polish space.

After obtaining these results and recalling the use of MLTS in other works (the counterexamples in D'Argenio et al. (2012); Wolovick (2012) are non-probabilistic NLMP), we conclude that these models provide a simple framework that can be considered as a first test scenario for conjectures about non-deterministic and probabilistic processes over continuous state spaces.

\section{Acknowledgements}

I want to thank Prof. Xavier Caicedo for a nice discussion concerning modal logics and for his Example 13. Also C. Areces and M. Campercholi pointed out several necessary references. I acknowledge the careful reading by both of the referees, and their comments, that changed drastically the way the results were presented; I realized that some of the passages in my original version were truly difficult to read, so thanks again. I would also 
like to thank José G. Mijares for some comments on descriptive-set-theoretical issues. Finally, I would like to express my gratitude to Prof. Pedro D’Argenio for a very instructive talk on process algebra.

\section{References}

Blackburn, P., Benthem, J.V. and Wolter, F. (2006). Handbook of Modal Logic, Studies in Logic and Practical Reasoning, volume 3, Elsevier Science Inc., New York, NY, USA.

Bolognesi, T. and Brinksma, E. (1987). Introduction to the ISO specification language LOTOS. Computer Networks 14 (1) 25-59.

Celayes, P. (2006). Procesos de Markov Etiquetados sobre Espacios de Borel Estándar, Master's thesis, FaMAF, Universidad Nacional de Córdoba.

Danos, V., Desharnais, J., Laviolette, F. and Panangaden, P. (2006). Bisimulation and cocongruence for probabilistic systems. Information and Computation 204 (4) 503-523.

D’Argenio, P., Wolovick, N., Sánchez Terraf, P. and Celayes, P. (2009). Nondeterministic labeled Markov processes: Bisimulations and logical characterization. In: QEST, IEEE Computer Society $11-20$.

D’Argenio, P.R., Sánchez Terraf, P. and Wolovick, N. (2012). Bisimulations for non-deterministic labelled Markov processes. Mathematical Structures in Computer Science 22 (1) 43-68.

Desharnais, J. (1999). Labeled Markov Process, Ph.D. thesis, McGill University.

Desharnais, J., Edalat, A. and Panangaden, P. (2002). Bisimulation for labelled Markov processes. Information and Computation 179 (2) 163-193.

Desharnais, J., Laviolette, F. and Turgeon, A. (2011). A logical duality for underspecified probabilistic systems. Information and Computation 209 (5) 850-871.

Doberkat, E.E. (2005). Stochastic relations: Congruences, bisimulations and the Hennessy-Milner theorem. SIAM Journal on Computing 35 (3) 590-626.

Doberkat, E.E. (2007a). Kleisli morphisms and randomized congruences for the Giry monad. Journal of Pure and Applied Algebra 211 (3) 638-664.

Doberkat, E.E. (2007b). Stochastic Relations: Foundations for Markov Transition Systems, Studies in Informatics Series, Chapman \& Hall/CRC, Taylor \& Francis.

Doberkat, E.E. (2009). Stochastic Coalgebraic Logic, Monographs in Theoretical Computer Science, Springer.

Edalat, A. (1999). Semi-pullbacks and bisimulation in categories of Markov processes. Mathematical Structures in Computer Science 9 (5) 523-543.

Kechris, A.S. (1994). Classical Descriptive Set Theory, Graduate Texts in Mathematics, volume 156, Springer-Verlag.

Kechris, A.S. (2011). Classical descriptive set theory; corrections and updates. Available at http://www.math. caltech.edu/papers/CDST-corrections.pdf.

Kracht, M. (1999). Tools and Techniques in Modal Logic, Studies in Logic and the Foundations of Mathematics, Elsevier.

Larsen, K.G. and Skou, A. (1991). Bisimulation through probabilistic testing. Information and Computation 94 (1) 1-28.

Moschovakis, Y.N. (2009). Descriptive Set Theory, 2nd edition, Mathematical Surveys and Monographs, American Mathematical Society.

Rutten, J.J.M.M. (2000). Universal coalgebra: A theory of systems. Theoretical Computer Science 249 (1) 3-80. 
Sánchez Terraf, P. (2011). Unprovability of the logical characterization of bisimulation. Information and Computation 209 (7) 1048-1056.

Scott, D. (1964). Invariant Borel sets. Fundamenta Mathematicae 56 (1) 117-128.

Srivastava, S.M., A Course on Borel Sets, Graduate Texts in Mathematics, volume 180, Springer.

Wolovick, N. (2012). Continuous Probability and Nondeterminism in Labeled Transition Systems, Ph.D. thesis, Universidad Nacional de Córdoba. 